\title{
Zwischen pädagogischer und geselliger Kommunikation - Eine ethnographische Annäherung an die Erforschung von Ungewissheit in hybriden Lernsettings
}

\author{
Karola Cafantaris ${ }^{1}$ \\ Angenommen: 4. Oktober 2021 / Online publiziert: 28. Oktober 2021 \\ (c) Der/die Autor(en) 2021
}

\section{Zusammenfassung}

Dieser Beitrag der Zeitschrift Gruppe. Interaktion. Organisation. (GIO) untersucht den Umgang mit Ungewissheit in einem hybriden Setting der Erwachsenenbildung. Der Artikel erläutert zunächst die zentralen Merkmale pädagogischer Ungewissheit in Zeiten zunehmender gesellschaftlicher Veränderung. Daraufhin werden die Bezüge pädagogisch gerahmter Gruppensettings zu der dargestellten Thematik erschlossen. Anhand der Erläuterung theoretischer Rahmenbegriffe wie Interaktion und Kommunikation sowie pädagogischer Kommunikation wird die empirische Analyse eines Fallbeispiels vorbereitet. Im Anschluss daran werden anhand von Ausschnitten aus einem ethnographischen Beobachtungsprotokoll exemplarisch Kommunikationsformate analysiert, die auf den Umgang mit Ungewissheit im hybriden Setting verweisen. Die Schlussbetrachtung nimmt eine Diskussion der Ergebnisse der empirischen Analyse vor und bettet diese in die im ersten Teil des Artikels erörterten theoretischen Bezüge ein.

Schlüsselwörter Interaktion · Ungewissheit · Hybride Lernsettings · Kommunikation · Pädagogische Kommunikation · Ethnographie

\section{Between pedagogic and informal communication-An ethnographic approach to exploring uncertainty in hybrid learning settings}

\begin{abstract}
This article in the journal Gruppe. Interaktion. Organisation. (GIO) elaborates how to deal with uncertainty in a hybrid setting of adult education. The article first explains the central characteristics of pedagogical uncertainty in times of increasing social change. Then, the references of pedagogically framed group settings to the presented topic are developed. By explaining theoretical framework terms such as interaction and communication as well as pedagogical communication, the empirical analysis of a case study is prepared. Subsequently, excerpts from an ethnographic observation protocol will be used to analyze exemplary communication formats that illustrate how uncertainty is dealt with in hybrid settings. The conclusion discusses the results of the empirical analysis and embeds them in the theoretical framework discussed in the first part of the article.
\end{abstract}

Keywords Interaction - Uncertainty $\cdot$ Hybrid learning settings $\cdot$ Communication $\cdot$ Pedagogical communication $\cdot$ Ethnography

Dr. Karola Cafantaris

karola.cafantaris@hsu-hh.de

1 Helmut-Schmidt-Universität Universität der Bundeswehr Hamburg, Hamburg, Deutschland

\section{Einleitung}

Ungewissheit, Unsicherheit und Risiko sind zentrale Begriffe, die das Leben in der Moderne zu beschreiben suchen (vgl. Helsper 2008). So gelte Ungewissheit als „Strukturmerkmal jeder Lebenspraxis“ (ebd., S. 162) und mit dem 
„Übergang zur Moderne“ (ebd.) sei „eine immer grundlegendere Aufstörung von Gewissheiten verbunden“ (ebd.). Dies berücksichtigend ist auch der Bereich der Bildung und Erziehung von dieser Gegenwartsdiagnose nicht ausgeschlossen. Bildungsinstitutionen und -einrichtungen sowie die Interaktionsprozesse, die in ihnen stattfinden, sind davon berührt und sehen sich fortlaufenden Reformierungsprozessen ausgesetzt, es werden Programme der Optimierung entwickelt und die Organisation von Bildung wird fortlaufend neu strukturiert. Pädagogisch formierte Lernund Bildungsorte sehen sich einem zunehmenden Veränderungs- und Hybridisierungsprozess ausgesetzt (vgl. Seitter 2011). Stichworte wie etwa die Digitalisierung von Lernwelten führen zu einer Veränderung der Orte des Lernens und der Bildung, die mit traditionellen Begrifflichkeiten kaum mehr zu beschreiben ist. Gleichzeitig gehen damit auch Veränderungen der Vermittlung und der Bereitstellung von Wissen einher. Längst sind die traditionellen Orte der Bildung und des Lernens nicht mehr die einzigen Orte, an denen Wissen institutionell vermittelt wird. So gewinnen rund um die Diskussion über das informelle Lernen diejenigen Orte an Bedeutung, die dem Bereich der Freizeitgestaltung oder dem privaten Kontext, etwa der Familie, zugeordnet werden. In Anbetracht der Hybridisierung von pädagogischen Lernorten, der Digitalisierung sowie der zunehmenden gesellschaftlichen Veränderung stehen altbekannte Konzeptualisierungen von Orten des Lernens auf dem Prüfstand und es entstehen neue Forschungsfragen, die einerseits Ungewissheit mit einbeziehen müssen und andererseits Möglichkeiten der Erschließung und Anknüpfung an Diskurse bieten sollen. Es geht also nicht mehr um die Frage, ob Ungewissheit dem pädagogischen Handeln innewohnt, sondern vielmehr wird die Frage relevant, wie in pädagogischen Interaktionen mit Ungewissheit umgegangen wird (vgl. Paseka et al. 2018).

Jochen Kade (2004) hat mit der Theorieperspektive der pädagogischen Kommunikation, der die Unterscheidung von Vermittlung und Aneignung (ebd., S. 205ff) zu Grunde gelegt wird, eine Theorie hervorgehoben, die die Ungewissheit pädagogischer Interaktionen miteinbezieht. So geht die Theorie pädagogischer Kommunikation davon aus, dass Wissensvermittlung nicht linear auf die Aneignung der Lernenden bezogen werden kann und die Unterscheidung von Vermittlung und Aneignung zum Bezugsproblem des ungewissen Verlaufs pädagogischer Operationen führt (vgl. ebd.). Die „kommunikative Aneignung“ (ebd., S. 208ff) schließlich sei deshalb eine „Erfindung pädagogischer Kommunikation“ (ebd., S. 209), um die ,unzugängliche Aneignung des psychischen Systems wenigstens in Form eines ausdeutbaren Derivats" (ebd.) in ihre Operationen zu integrieren. Die „Unmöglichkeit von Erziehung“ (ebd.) würde so „in deren Unwahrscheinlichkeit transformiert“ (ebd.).
Gleichzeitig wird durch diese kommunikationstheoretische Modellierung pädagogische Kommunikation losgelöst von den einschließlich dafür vorgesehenen Institutionen, auch an den Rändern des Pädagogischen, beobachtbar. Die erziehungswissenschaftliche Auseinandersetzung stellt sich unter dem Stichwort der „Universalisierung des Pädagogischen“ (vgl. Lüders et al. 2004) schon seit Längerem die Frage nach der Expansion und Verbreitung pädagogischer Denk- und Handlungsstrukturen. Mit dieser Idee der Universalisierung geht gleichzeitig eine Veränderung pädagogischer Angebotstypen wie auch ihrer Erforschung einher, der es hauptsächlich darum geht, einen „Überblick über die Pluralität von Bildungssituationen zu gewinnen“ (Seitter 2000, S. 16). Die Erforschung der Universalisierung des Pädagogischen erfordert demzufolge neben der theoretischen Auseinandersetzung mit dem Lernen im Erwachsenenalter (vgl. dazu Dinkelaker 2018) methodologische Neubestimmungen und Positionierungen innerhalb der Forschungsperspektiven, die sich mit Fragen nach dem Verhältnis von Ungewissheit und der „Ungewissheitsabsorption“ (Luhmann 2000, S. 186) in pädagogischen Settings beschäftigen. Zieht man zu diesen Überlegungen die Organisationstheorie Niklas Luhmanns hinzu, lässt sich Unsicherheit als Differenz von Wissen und Nichtwissen fassen (vgl. ebd.). Unsicherheit sei dabei nicht wie ,im alltäglichen Sprachgebrauch [...] als ein dysfunktionaler Zustand zu begreifen“, sondern „fortbestehende und immer wieder neu generierte Unsicherheit“" sei die ,wichtigste Ressource der Autopoiesis des Systems“ (ebd., S. 185). Da ohne Unsicherheit keine Entscheidungen mehr getroffen werden müssten, fände die Organisation ,im Zustande kompletter Selbstfestlegung ihr Ende und würde mangels Tätigkeit aufhören zu existieren“ (ebd.). So sei Ungewissheit die „Prämisse von Organisationen“ und ihr Erfolg läge ,in der Behandlung dieser Ungewissheit“" (ebd., S. 10).

Für pädagogische Settings nimmt die Auseinandersetzung mit der Frage von Ungewissheit aufgrund der zunehmenden Hybridisierung des Lernens und der Bildung zu. Zwar gingen Erziehungswissenschaft und Pädagogik in der Regel von „reinen Strukturen“ (Dinkelaker 2008, S. 20) der Ausgestaltung und Formierung von Bildungsorten aus, in denen der Umgang mit Wissen „lebensphasen- oder organisationsorientiert gedacht“" (ebd.) würde. Dem stehen aber Ansätze entgegen, die zwischen formalen und nichtformalisierten Lern- und Bildungssettings zunehmend verschwimmende Trennlinien sehen und vermehrt ,Versuche [...] beobachten, diese scharfe Trennung zu überwinden“(ebd.). Aufbauend auf dem Konzept des lebenslangen Lernens hat sich ein Forschungsstrang etabliert, der gezielt hybride Lernorte untersucht (vgl. u. a. Dinkelaker 2008; Kade und Seitter 2007).

Die hier aufgezeigten Bezüge erlauben es nun, einerseits von der Kontingenz pädagogischer Vermittlungs- und 
Aneignungsprozesse auszugehen und andererseits pädagogische Formationen nicht nur an den traditionell dafür vorgesehenen Orten zu erwarten, sondern den Blick auch für andere Interaktionszusammenhänge und ihre mögliche pädagogische Strukturierung zu öffnen. In diesem Beitrag möchte ich daran anknüpfen und anhand eines ethnographischen Beobachtungsprotokolls, das im Rahmen der Beobachtung eines hybriden Lernsettings entstanden ist, den pädagogischen Umgang mit Ungewissheit empirisch einholen. Die Ethnographie als Methode der Erziehungs- und Sozialwissenschaften eignet sich für solch ein Vorgehen, da ihr „weicher“ Methoden- und ihr „harter“ Empiriebegriff (vgl. Amann und Hirschauer 1997, S. 9) es ermöglicht, ebendiesen Untersuchungsorten mit einer ,empirischen wie theoretischen Neugier“ (ebd.) zu begegnen. Ethnographische Untersuchungen hybrider Lernsettings erlauben es demzufolge, die vorgefundene Ungewissheit als solche zum Thema der Forschung zu machen und die vielfältigen Aneignungsweisen von Wissen zu thematisieren (vgl. Wilde 2015; Cafantaris 2020). Diese Überlegungen führen mich alsdann zu der Frage, wie in einem hybriden Lernsetting interaktional mit pädagogischer Ungewissheit umgegangen wird. Um dieser Frage nachzugehen, gliedert sich der Beitrag in folgende Abschnitte: Zunächst sollen die Bedingungen von Kommunikation unter Anwesenden (vgl. Kieserling 1999) spezifiziert werden (2). Anschließend wird die ethnographische Forschungshaltung skizziert, um sodann eine Analyse zweier Ausschnitte aus einem Beobachtungsprotokoll vorzunehmen (3). Die Ergebnisse der Analyse werden abschließend einer Schlussbetrachtung unterzogen und im Hinblick auf ausstehende Forschungsvorhaben diskutiert (4).

\section{Die Erforschung von Interaktion unter Berücksichtigung von Ungewissheit}

Die Untersuchung interaktionaler Zusammenhänge gilt als interdisziplinär verortetes Forschungsfeld. Um nur einen ausschnitthaften Überblick zu geben, handelt es sich etwa im Bereich der Soziologie um Forschungen, die zum Beispiel den Bereich der sozialen Beziehung oder der Netzwerkbildung betreffen (vgl. Vonneilich 2020). Psychologische Ansätze behandeln demgegenüber unter sozialpsychologischen Gesichtspunkten unter anderem Fragen der sozialen Interaktion, der Gruppensozialisation und Gruppenidentifikation (vgl. Stürmer und Siem 2020), wohingegen der pädagogische Blick Fragen zu Lern- und Bildungsprozessen in interaktionalen Zusammenhängen zum Beispiel unter dem Begriff der Lerngruppe, Schulklasse oder etwa über das Konzept der Peerkommunikation zu fassen sucht (vgl. Breidenstein und Kelle 1998, 2002). Vor allem im Hinblick auf die Untersuchung hybrider Lernsettings werden interaktionale Bezugnahmen zentral gestellt, da sie Auskunft über den Formalisierungsgrad des jeweiligen Feldes zu geben vermögen. Deshalb scheint es mir im Hinblick auf die Vorbereitung der Analyse eines Beobachtungsprotokolls relevant zu sein, zu diskutieren, wie sich Interaktion als „Kommunikation unter Anwesenden“ (Kieserling 1999) fassen lässt. Dem voranzustellen ist die an Erving Goffman (1959) anschließende Überlegung zu sozialen Gruppen als Interaktionszusammenhang, in dem die Gruppenmitglieder als rollenförmig und aufeinander abgestimmt gelten (vgl. ebd., S. 12f.) und weniger die Gefühle des Einzelnen, sondern vielmehr die Regeln und Strukturen der Gruppe relevant für die Interaktion sind. Dementsprechend beschreibt Goffman (ebd.) die Abstimmung der Gefühle von Einzelnen auf die geteilten Annahmen einer Gruppe und der anderen Gruppenmitglieder.

Damit soll nicht gesagt sein, es bestehe jene Übereinstimmung, die sich einstellt, wenn der Einzelne offen das ausdrückt, was er wirklich fühlt, und mit den Gefühlen der anderen ehrlich übereinstimmt. Diese Art von Harmonie ist ein optimistisches Ideal und jedenfalls nicht unbedingt notwendig für das reibungslose Funktionieren der Gesellschaft. Man erwartet im Gegenteil von jedem Teilnehmer, daß er seine unmittelbaren tieferen Gefühle unterdrückt und einen Aspekt der Situation ausdrückt, den seiner Ansicht nach die anderen wenigstens vorübergehend akzeptieren können. Diese oberflächliche Übereinstimmung, die den Anstrich von Einigkeit hat, wird ohne Schwierigkeiten aufrechterhalten, wenn jeder seine eigenen Bedürfnisse hinter der Verteidigung von Werten verbirgt, denen sich alle Anwesenden verpflichtet fühlen. (Ebd.)

Auf diese Weise entsteht laut Goffman „eine Art von Modus vivendi innerhalb der Interaktion“ (ebd., S. 13). Das heißt, die Gruppenmitglieder tragen ,gemeinsam zu einer umfassenden Bestimmung der Situation“ bei, die ,weniger auf echter Übereinstimmung über die Realität" beruht als ,,auf echter Übereinstimmung darüber, wessen Ansprüche in welchen Fragen vorläufig anerkannt werden" sollten, und meist auch darüber, „daß es wünschenswert ist, einen offenen Konflikt zwischen widersprechenden Bestimmungen der Situation zu vermeiden“ (ebd.). Kieserlings (1999) Überlegungen hinzuziehend, gilt als „Konstitutionsprinzip von Interaktion [...] seither das Erfordernis der gemeinsamen Anwesenheit" (ebd., S. 18). Seine kommunikationstheoretisch gerahmte Modellierung des Interaktionsbegriffs nimmt diese Setzung auf und beschreibt mit „Kommunikation unter Anwesenden“ (ebd., S. 24 ff.) eine Form der Kommunikation, die sich von anderen Formen der Kommunikation unterscheide, die ohne die „Erfordernis der gemeinsamen Anwesenheit" (ebd.) auskommen und dadurch „mehr Möglichkeiten habe[n], aber auch unter spezifischer 
definierte Beschränkungen gestellt werden können“ (ebd.). Ein zentrales Merkmal der „Undifferenziertheit von Interaktion“ (ebd., S. 37) sieht Kieserling darin, dass es in Interaktionen ,nur ein Zentrum der Konvergenz von Aufmerksamkeit geben“ (ebd.) könne. Dies gelte vor allem deshalb, „weil Interaktionen Kommunikationsprozesse durchführen und sich auf dafür geeignete Themen beziehen müssen" (ebd.). Diese Konzentration von Interaktionen auf jeweils ein Thema nennt Kieserling „Zwang zur Serialität“ (ebd., S. 27).

Richtet man nun den Blick von der Interaktion auf Kommunikation, bietet sich der Kommunikationsbegriff Luhmanns an, welcher Kommunikationsprozesse von Bewusstseinsprozessen abkoppelt. Luhmann beschreibt Kommunikation als ,eine emergente Realität, ein[en] Sachverhalt sui generis“, die zustande komme „durch die Synthese von drei verschiedenen Selektionen - nämlich Selektion einer Information, Selektion der Mitteilung dieser Information und selektives Verstehen oder Mißverstehen dieser Mitteilung und ihrer Information“ (Luhmann 2005, S. 111). Dies berücksichtigend, führe Kommunikation zur ,Zuspitzung der Frage, ob die mitgeteilte und verstandene Information angenommen oder abgelehnt" (ebd., S. 115) werde. Kommunikation beinhaltet dementsprechend immer auch die Ungewissheit, ob das Kommunikationsangebot abgelehnt oder angenommen wird. Angesichts dessen ist laut Luhmann ,alle Kommunikation riskant" (ebd.). Die damit beschriebene Ungewissheit von Kommunikation wird im Begriff der Kontingenz oder auch der ,doppelte[n] Kontingenz“ (Parsons und Shils 1951, S. 3-29; zitiert nach Meseth et al. 2011, S. 223) verdeutlicht. In Anbetracht des Bezugsproblems, dass an Kommunikation gleichsam „beliebig“ angeschlossen werden kann, wird die Frage virulent, „wie soziale Ordnung operativ möglich“ (Meseth et al. 2011, S. 224) ist und wie sich lose gerahmte Kommunikation dennoch themenzentriert vollziehen kann. So könne man ,jenes Moment der Offenheit [auch] als doppelte Kontingenz analysieren: Jeder kann nicht nur so handeln, wie es der andere erwartet, sondern auch anders, und beide stellen diese Doppelung in erwartete und andere Möglichkeiten an sich selbst und am anderen in Rechnung" (Kieserling 1999, S. 87).

Auch pädagogisch gerahmte Operationen der Vermittlung und Aneignung von Wissen können deshalb nur in ihrer kommunikativen Emergenz sichtbar werden - und zuletzt auch nur durch die dritte Sequenzstelle des Verstehens und deren kommunikative Anzeige überprüfbar gemacht werden (vgl. Kade 2017). Im Hinblick auf pädagogisch formierte Settings tritt pädagogische Kommunikation (Kade 2004, 2017) als Prozessierung der Differenz von Vermittlung und Aneignung als hervortretendes Kommunikationsformat in den empirischen und theoretischen Fokus. Das „Konzept der pädagogischen Kommunikation“ ist laut Kade
(2017, S. 4) „ein Konzept direkter pädagogischer Strukturierung von Aneignung von Wissen und Werten. Operativ grundlegend ist zunächst die Differenzierung zwischen den aufeinander bezogenen Elementen Vermitteln, Wissen, Aneignen und Überprüfen/Bewerten“ (ebd.). Außerhalb des schulischen Unterrichts ist pädagogische Kommunikation zudem eher „flüchtig“, als dass sie verdichtet auftritt. Mit Blick darauf sind vor allem die Ausführungen von Kade und Seitter (2007) zu nennen, die in Settings, in denen pädagogische Kommunikation auftritt, ,hybrid[e]“ (ebd., S. 76) Formen der Kommunikation verzeichnen. Wissensvermittlung alterniere hierbei immerzu mit anderen kommunikativen Formen (vgl. ebd.). Zum Problem werde die „Polyfunktionalität, Uneindeutigkeit“" (ebd.) dann, wenn es um die Frage gehe, wie sich „Wissensvermittlung überhaupt als Form etablieren und behaupten" (ebd.) könne, da aneignungsbezogene Kommunikationsformate in hybriden Settings stärker mit anderen kommunikativen Formaten konkurrierten als in formalisierten Settings (vgl. ebd.).

Wie lassen sich diese Kommunikationsformate beispielhaft bestimmen? Wie im folgenden Kapitel anhand empirischen Materials dargelegt werden wird, können im hybriden Setting hauptsächlich drei Kommunikationsformate beobachtet werden. Gesellige Kommunikation stellt eine Form von Kommunikation unter Anwesenden dar, die nicht ausschließlich auf ein Thema bezogen ist und daher durchaus als die Bedingung für Störungen innerhalb themenzentrierter Kommunikationsphasen gefasst werden kann (vgl. Seitter 2000, S. 92). Einfache Wissensvermittlung liegt laut Kade (2017) dann vor, wenn Wissen unter Absehung des Aspekts der Aneignung vermittelt wird, wie es etwa bei Vorträgen oder Fernsehsendungen der Fall ist, in denen Wissensvermittlung unvermeidbar mitläuft (vgl. ebd., S. 6). Hingegen sei für pädagogische Kommunikation die Differenz zwischen Vermittlung und Aneignung ,mehr als eine bloße, der Sache nach ohne Konsequenz bleibende Spezifizierung eines allgemeinen systemtheoretischen Kommunikationsbegriffs" (ebd., S. 4). Entscheidend sei in pädagogischen Settings vielmehr die „Umwandlung des Konzepts der Kommunikation (als Synthese der Elemente Information, Mitteilen und Verstehen) in ein intentional gerichtetes, operativ eng verzahntes erziehungswissenschaftliches (damit auch normatives) Konzept pädagogischer Kommunikation“ (ebd.).

\section{Ethnographische Annäherungen}

Die Ethnographie gilt mittlerweile als ,zentrale Strategie empirischer Forschung" (Breidenstein 2017, S. 10). Fokussiert auf erwachsenenpädagogische Settings sind neben Untersuchungen von sozialpädagogischen Feldern (u. a. Hünersdorf 2009) und non-formalen Bildungsorten wie dem 
Vereinswesen (u.a. Seitter 1999) ebenso Untersuchungen zu informellen Orten des Lernens von Erwachsenen (u.a. Wilde 2015; Cafantaris 2020) zu verzeichnen. Es handelt sich um qualitativ ausgerichtete Studien, die in ,der eigenen Gesellschaft kulturelle Praktiken von sozialen Gruppen in deren unmittelbarer Umgebung untersuch[en] und Sinnzusammenhänge aus der Perspektive der Untersuchten nachzuvollziehen versuch[en]" (Schulz 2014, S. 225). Die methodologische Prämisse der ethnographischen Haltung ist die ,temporäre Mitgliedschaft der Forschenden im Kreis der Beforschten“ (ebd.), die als „kompetente Interpreten der sozialen Welt“ (ebd.) verstanden werden. Der Ethnographie geht es in einem weiten Verständnis darum, die feldeigenen Logiken durch das eigene Erleben zu durchleuchten und die im Feld zu beobachtenden Handlungspraktiken in der eigenen Aneignung zu reflektieren. Der Durchführung des Feldaufenthalts, der beobachtenden Haltung und der anschließenden Protokollierung des Erlebten liegen zahlreiche forschungspraktische Auseinandersetzungen zugrunde (vgl. Amann und Hirschauer 1997; Breidenstein et al. 2013; Geertz 1987). Die Forschenden sind aufgefordert, sich gegenüber dem zu untersuchenden Feld künstlich auf Distanz zu bringen (vgl. Amann und Hirschauer 1997) und mit einem möglichst offenen Blick an das Feld heranzutreten. Ebenso wie einer offenen Forschungshaltung wird dem Eintritt ins Feld eine große Bedeutung zugeschrieben, der dazu verhilft, die Strukturen des Untersuchungsfelds unter einer forschungsbezogenen Fragestellung vertiefend in den Blick zu nehmen. Die teilnehmende Beobachtung stellt dabei eine spezifische Form sozialer Interaktion dar (vgl. Cafantaris 2021), bei der die Reziprozität zwischen Beobachtenden und Beobachtetem ,zum zentralen methodologischen Problem“ (Lindner 1981, S. 51) wird. Im Beobachtungsprotokoll findet schlussendlich eine Art finalisierter Archivierung des Erlebten statt, die jedoch mitnichten als statisch zu begreifen ist (vgl. Cloos 2010). Es handelt sich vielmehr um eine Zusammenschau der im Feld erhobenen Daten und begleitet die Forschenden in gewisser Weise in den Schritten nach der Erhebung, vor allem bei der abschließenden theoretischen Rückbindung des erhobenen Materials, an (vgl. ebd.).

Die vorangegangenen Überlegungen sollen nun anhand empirischen Materials aus einer Untersuchung eines Geschichtsprojekts für Erwachsene verdeutlicht werden.

Die im Folgenden analysierten Beobachtungsprotokollausschnitte sind im Kontext einer ethnographischen Untersuchung entstanden, auf der die Studie Umgang mit Wissen in der Kulturarbeit für Erwachsene. Pädagogische Kommunikation in hybriden Settings der Erwachsenenbildung (Cafantaris 2020) basiert. Das beforschte
Stiftungsprojekt „GeschichteErforschen“1 ist ein in regelmäßigen Abständen stattfindendes ehrenamtliches Projekt, das 25 Bürgerinnen und Bürgern eine 18-monatige Auseinandersetzung mit einem stadtteilhistorischen Thema ermöglicht. Dabei werden die Teilnehmenden durch eine eng geknüpfte Projektstruktur, die Projektkoordination und einen finanziellen Beitrag unterstützt. Am Ende der Projektlaufzeit soll ein Produkt zu der jeweiligen Themenbearbeitung entstehen. Es finden hauptsächlich drei Veranstaltungsformate innerhalb der Projektstruktur statt: die Staffelübergabe als Initiations- und Verabschiedungsritual, das Werkstatt-Treffen als wissensvermittlungsbezogener Workshop und die informellen Treffen, die einmal pro Monat den regelmäßigen Austausch über die jeweiligen Projekte ermöglichen sollen.

In der ausgewählten Szene aus einem der informellen Treffen soll gezeigt werden, dass für die Systembildung dieses Geschichtsprojekts drei Kommunikationsformate funktional sind: gesellige Kommunikation, Wissensvermittlungskommunikation und pädagogische Kommunikation. Vor allem die besondere Funktionalität geselliger Kommunikation im Hinblick auf die diverse Zusammensetzung der Gruppe bezüglich des Alters und insbesondere der verschieden gelagerten Wissenshaushalte wird in der Analyse herausgestellt. Das Ausbalancieren zwischen individuellen Entfaltungsprozessen und gruppenbezogenen Homogenisierungsbestrebungen kann indessen als Bezugsproblem des hybriden Settings markiert werden, das im kommunikativen Vollzug des informellen Treffens fortlaufend bearbeitet werden muss. Während pädagogische Kommunikation und Wissensvermittlung die aneignungsbezogenen Sequenzen der Kommunikation strukturieren, fungiert gesellige Kommunikation vielmehr als kontinuitäts- und stabilitätsgenerierender Faktor innerhalb der Gruppe und stellt einen Umgang mit der Heterogenität der Teilnehmenden innerhalb der Treffen dar (vgl. Cafantaris 2020).

\subsection{Erster Ausschnitt aus dem Beobachtungsprotokoll: Formierung der Kommunikation}

Im ersten Ausschnitt, der an dieser Stelle nur zusammengefasst werden soll, beschreibt das protokollierende Ich das Aufeinandertreffen zweier sich bereits bekannter Personen. Es handelt sich um einen Teilnehmer des untersuchten Geschichtsprojekts, den die protokollierende Person - aufgrund des bereits intensiveren Kontakts, der auf mehreren vorangegangenen Interviewsessions beruht - als gleichsam Vertrauten innerhalb des Projektkontextes beschreibt. Die

\footnotetext{
${ }^{1}$ Die wissenschaftliche Begleitung des Projekts „GeschichteErforschen“ (2012-2014) erfolgte unter der Leitung von Prof. Dr. Wolfgang Meseth.
} 
beiden Personen, so das Protokoll, suchen gemeinsam den konkreten Ort, an dem die Veranstaltung stattfinden soll und kommen knapp bemessen, jedoch pünktlich an. Das Treffen findet in einem historischen Weinkeller statt. Der Ort wird als bereits eingerichtet beschrieben, so sind Sitzmöglichkeiten hergerichtet und es ist Verpflegung für die Teilnehmenden auf dem Tisch bereitgestellt. Die Protokollantin und der Teilnehmer nehmen auf den Sitzen Platz und warten auf den offiziellen Auftakt des Treffens. Wie sich in dem nun folgenden Abschnitt zeigt, wird durch die vom Leiter des Treffens vorgenommene Veranstaltungseröffnung bereits zu Anfang eine Stimmung erzeugt, die den Fokus auf ein themenzentriertes Gespräch lenkt. Dieser durch die pädagogische Leitung und das vorbereitete Setting angedachte vorstrukturierte Ablauf des Treffens wird jedoch im weiteren Verlauf durch gesellige Sequenzen unterbrochen, wodurch die thematischen An- und Ausschlüsse diffundierenden Charakter haben.

Der Projektkoordinator sagt, dass es 19:00 Uhr ist und dass er dann mal anfängt, es würden sicherlich einige zu spät dazukommen. Er kommt schnell zur Sache und macht schon eingangs klar, dass einige Punkte besprochen werden sollten. [...] Der Projektkoordinator fängt mit der Ankündigung an, dass das nächste informelle Treffen im Juli aufgrund der Sommerferien nicht stattfindet. In diesem Zusammenhang rät er den Teilnehmenden, ihr Projekt auch mal liegen zu lassen über den Sommer. Das hätte positive Effekte, u.a. dass man im Nachhinein überrascht darüber sei, was man schon alles geschafft hat. Dann wird der organisatorische Hinweis gegeben, dass das nächste Treffen am letzten Dienstag im August im Stadtteil [Name des Stadtteils] stattfindet. Als weitere Ankündigung berichtet der Projektkoordinator von einer Open-air-Ausstellung auf einem sehr zentral gelegenen Platz der Stadt. Diese Ausstellung würde die Möglichkeit bieten, sein Projekt an einem öffentlichen Platz zu präsentieren. In Glaskästen könnte man nach eigenem Ermessen sein Projekt darstellen. Einer der Teilnehmenden fragt, wann das denn sei, der Projektkoordinator antwortet fast schon mit einem Lachen, dass diese Ausstellung erst in etwas weniger als einem Jahr geplant ist. Er sagt außerdem, dass mindestens zehn TeilnehmerInnen mitmachen müssen, um die Ausstellung auf die Beine stellen zu können, und dass sie wichtig wäre, um auf die nächste Staffel des Projekts „GeschichteErforschen“ aufmerksam zu machen: „Wir würden das gerne machen - auch, um auf die 5. Staffel hinzuweisen. Zudem sind die Plakatwände eine vorzügliche Gelegenheit, sich der Stadtgesellschaft zu präsentieren.“ Dennoch sei das, was präsentiert wird, auf eigene Kosten herzustellen. Es sei zwar eine Menge Arbeit, würde aber auch viel Spaß machen. (Beobachtungsprotokoll_6. IT)

\subsubsection{Analyse}

Beschrieben wird zunächst ein formaler Akt, der aus unterschiedlichen (pädagogischen) Settings als Einstiegssequenz bekannt erscheint. Es werden „Ankündigungen“ unterschiedlicher Art vorgenommen, die die Organisation der weiteren informellen Treffen wie auch die Arbeit an den jeweiligen Projekten betreffen. Auffällig erscheint zunächst das stringente Aufzählen von Terminen durch den Veranstaltungsleiter. So würde man bei einem informellen Treffen wohl zunächst eher ein geselliges Parlieren erwarten, das sich erst langsam hin zu einer themenzentrierten Kommunikation entwickelt. Die Beobachterin nimmt im Gegensatz dazu jedoch vielmehr die Auflistung von Terminen wahr. $\mathrm{Zu}$ einer Nachfrage eines Teilnehmers kommt es erst, nachdem der Projektkoordinator seine Rede beendet hat. Diese Ausrichtung auf den zeitlichen Verlauf des Projekts adressiert die Gruppe als ein für den genannten Zeitraum formiertes Kollektiv, das im Sinne einer Gemeinschaft über bereits ausgehandelte Regeln und Aufgaben, etwa die Arbeit an einem Projekt (,,rät er den Teilnehmenden, ihr Projekt auch mal liegen zu lassen über den Sommer") und ebenso über gemeinsame Ziele verfügt, wie die anstehende Ausrichtung einer gemeinsamen Ausstellung zeigt. Auch erscheint die Reaktion des Teilnehmers, nach dem genauen Termin der Ausstellung zu fragen, als Verständnisfrage, die potenziell alle Teilnehmenden interessieren könnte. Der Teilnehmer nimmt eine Art Stellvertreterposition ein, indem er diese Information in der Öffentlichkeit für alle Gruppenmitglieder einholt. Das Lachen des Projektkoordinators und seine Beantwortung der Frage des Teilnehmers mit dem Verweis auf den noch lange dauernden Zeitraum, bis die Ausstellung tatsächlich stattfinden wird, rekurrieren erstens auf die Gewährleistung einer Vorlaufzeit, die den Teilnehmenden des Projekts eingeräumt wird und es ihnen möglicherweise erleichtern soll, ihren Ausstellungsgegenstand in angemessener Weise herzustellen. Zweitens und im Hinblick auf die weiteren protokollierten Ausführungen des Projektkoordinators geht es nicht nur um das laufende Geschichtsprojekt, sondern ebenso um die noch folgenden Projektstaffeln (,,auch, um auf die 5. Staffel hinzuweisen“). Die Ebene der Organisation (Stiftung) tritt an dieser Stelle kommunikativ in den Kontext des Treffens ein, indem der Projektkoordinator auf die Stabilisierungs- und Präsentationsfunktion der Ausstellung für das Geschichtsprojekt hinweist. Zusammenfassend stellt sich die interaktionale Bezugnahme beim hier dargestellten Auftakt des Treffens wie folgt dar: Über die Vermittlung von auf die Organisation und Planung zukünftiger Treffen und der Ausstellung bezogenen 
Informationen und den Hinweis, wie über den Sommer mit den jeweiligen Einzelprojekten umgegangen werden soll, wird bereits zu Beginn des Treffens eine thematische Rahmung in Bezug auf die Ausstellung eingeführt. Durch das Aufrufen von Terminen und die damit verbundene Werbung von Personen, die sich an der Ausstellung beteiligen möchten, wird die Kontinuität des Projektkontextes hergestellt. Gleichzeitig wird die terminliche Rahmung als auf die Zeit bezogene Orientierung für zukünftiges Handeln relevant. Außerdem bietet die Ausstellung den Teilnehmenden einen Ort der Präsentation des Arbeitsprozesses an ihrem Geschichtsprojekt.

\subsection{Zweiter Ausschnitt aus dem Beobachtungsprotokoll: Zwischen thema- tischer Zentrierung und Geselligkeit}

Eine Teilnehmerin meldet sich und bezieht ihren Redebeitrag auf die zu Anfang erwähnte Ausstellung. Sie sagt, dass sie es toll fände, wenn man der Ausstellung eine gemeinsame Klammer geben könnte. Sie denke dabei an die oft besprochene Frage, was ,unsere“ Stadt schön macht. Die neu Dazugekommenen wissen nicht, um welche Ausstellung es sich handelt, und fragen laut: „Welche Ausstellung?“ „Ausstellung - wer zahlt das?“

Der Projektkoordinator reagiert zunächst auf die Anfragen der Dazugekommenen und nennt auch geduldig die weiteren Ankündigungen, die diese nicht mitgehört hatten, da sie zu spät gekommen sind. Er berichtet außerdem von dem Alumni-Treffen an einem bekannten Ausflugsort und berichtet, dass dieses Treffen von der Stiftung sehr schön ausgerichtet wird. Es gäbe vorzügliches Essen und ein tolles Programm. Außerdem sei man kein richtiger Bewohner dieser Stadt [Name der Stadt] ${ }^{2}$, wenn man nicht einmal an diesem Ort übernachtet hätte: Gelächter und ich lache mit. Eine etwas ältere Teilnehmerin fragt, ob man denn dann in Schlafsälen schlafen müsste. Darauf hätte sie nicht sonderlich Lust. Der Projektkoordinator versucht sie zu beschwichtigen (...). Dann wendet er sich der Bemerkung der Teilnehmerin mit der Idee der ,gemeinsamen Klammer“ zu. [...] Der Projektkoordinator bezieht sich auf ihren Vorschlag, indem er ihre Idee annimmt und sie umwandelt zu einer neuen Idee. Er sagt, man könne zusätzlich zu den einzelnen Projektstellwänden eine Stellwand machen, auf

\footnotetext{
2 An dieser Stelle nennt der Projektkoordinator den Namen der Stadt und deren Bewohner (wie z. B. „Berliner“ oder „Hamburgerin“), was $\mathrm{zu}$ Anonymisierungszwecken geändert wurde. Die Aussage ist damit zu begründen, dass es sich bei diesem Ausflugsziel um einen Ort handelt, der des Öfteren von Schulklassen bei Klassenfahrten besucht wird.
}

der das Thema „Was [Name der Stadt] schön macht“ aus Sicht der einzelnen Projekte aufgegriffen wird. (Beobachtungsprotokoll_6.IT)

\subsubsection{Analyse}

Der zweite Ausschnitt aus dem Protokoll beginnt mit einer sachlich auf die geplante Ausstellung bezogenen Anmerkung einer Teilnehmerin, im Rahmen derer der Vorschlag geäußert wird, der Ausstellung einen gemeinsamen thematischen Rahmen zu geben. Das Protokoll verweist daraufhin auf verspätet bei der Veranstaltung eingetroffene Teilnehmende (,Dazugekommene“), die ebenso in das Thema der Besprechung eingeführt werden wollen. Anstatt nun auf die Anmerkung der Teilnehmerin einzugehen, werden zunächst die „Dazugekommenen“ in den Kontext des Themas Ausstellung eingeführt und über die weiteren Ankündigungen informiert. Den immerfort wiederholten Ankündigungen kommt ein zentrales Motiv innerhalb des Beobachtungsprotokolls zu. So durchläuft die Kommunikation gleichsam thematische Schleifen, die an der einen oder anderen Stelle wieder in Ausführungen zu den Ankündigungen münden und so die zuweilen gesellige Kommunikation wieder in eine themenzentrierte Kommunikation überführen. Die folgende Passage, die sich mit einem gemeinsamen Besuch eines bekannten Ausflugsziels beschäftigt, nimmt noch einmal den Bezugspunkt der Zeit auf. Erstens werden die Teilnehmenden als zukünftige „Alumni“ adressiert, die bei diesem Besuch erfolgreich das Projekt durchlaufen haben werden und deshalb dann nicht mehr nur Teilnehmende sind, sondern der zeitlich nunmehr unbegrenzten Gemeinschaft der Alumni angehören. Zweitens werden sie als Teilnehmende einer auf „Freizeit“ bezogenen Veranstaltung adressiert. Somit wird die Erwartungshaltung etabliert, dass die Teilnehmenden bis dahin ihre Projekte beendet haben werden. Die damit einhergehenden humoristischen Ausführungen der Teilnehmerin sowie des Projektkoordinators führen $\mathrm{zu}$ geselliger Zerstreuung der Kommunikation. Während die Kommunikation bei einer geselligen Zusammenkunft mit großer Wahrscheinlichkeit nun etwa in Einzelgespräche oder andere Formen diffuser Kommunikation übergegangen wäre, wird die Kommunikation im hybriden Setting durch die wiederholte Einholung der gemeinsamen thematischen „Klammer“ durch den Projektkoordinator wieder auf ein projektbezogenes Thema zentriert, indem er auf den inhaltlichen Vorschlag der Teilnehmerin eingeht. Er nimmt ihre Idee auf und wendet sie produktiv im Hinblick auf den Projektkontext und die Ausgestaltung der Ausstellung. 


\section{Zusammenfassung und Diskussion}

Bezieht man die Frage nach dem Umgang mit Ungewissheit in die zusammenfassende Analyse mit ein, verweist die hier beobachtete Kommunikation unter Anwesenden auf ein Wechselspiel von Öffnung und Schließung themenzentrierter Phasen und Formen der geselligen Zerstreuung (vgl. Cafantaris 2020, S. 118). Die Analyse verdeutlicht das komplexe Arrangement kommunikativer Prozesse, das beim Umgang mit Wissen in einem hybriden Setting entsteht. Unter der Bedingung von lose gekoppelten Anschlüssen der Kommunikation tritt die Ungewissheit über den Verlauf des Treffens und die thematischen Verdichtungs(un)möglichkeiten in Form von Zentrierung und Zerstreuung zunehmend als Bezugsproblem des hybriden Settings auf. Wie wird dieses Bezugsproblem im operativen Vollzug des Treffens bearbeitet? Die Teilnehmenden erscheinen im Protokoll als im zeitlichen Verlauf des Projekts „eingespielte“ Gruppe, die die Regeln der Interaktion bereits ausreichend ausgehandelt hat und sowohl organisatorische als auch thematische Verdichtungsmomente entstehen lassen kann. Trotz der diversen Voraussetzungen der Teilnehmenden wird im Zusammenspiel von Projektkoordination, räumlicher Vorstrukturierung und der Eigenverantwortlichkeit der Teilnehmenden ein Setting kreiert, das neben thematisch zentrierten Kommunikationsformaten auch gesellige Kommunikation zulässt. Und mehr noch liegt gerade die besondere Funktion geselliger Kommunikation auf der Hand: Sie bietet allen Teilnehmenden - ob historisch versiert oder nicht - kommunikativen Anschluss sowie Themen, an denen sie partizipieren können. Gesellige Kommunikation fungiert dabei als kontinuitäts- und stabilitätsgenerierender Faktor innerhalb der Gruppe und bearbeitet die Heterogenität der Teilnehmenden der formalen sowie informellen Zusammenkünfte auf der sozialen Ebene. Pädagogische Kommunikation entsteht innerhalb des Verhältnisses zwischen der externen Erwartung, an der angekündigten Ausstellung teilzunehmen und der Selbstbeobachtung der Teilnehmenden in Bezug auf ihr eigenes Projekt. Die thematische Verhandlung der geplanten Ausstellung ist indessen ein die Kommunikation und Interaktion stabilisierender Faktor für das Geschichtsprojekt. Während die themenbezogene Kommunikation eine gleichsam erwartbare thematische Verdichtung der Settings darstellt, tritt gesellige Kommunikation - neben pädagogischer und Wissensvermittlungskommunikation - weniger als diffundierende Variable in die Kommunikation ein, sondern in ihr werden allenfalls inhaltliche Verdichtungen aufgelöst, um an anderer Stelle wieder in die Themenzentrierung zu münden. Abschließend gilt es nach wie vor weiter zu verfolgen, wie die zunehmende Hybridisierung von Lernorten sowohl zukünftige pädagogische Formbildungen beeinflussen wird, als auch wie deren Erforschung etwa durch die Digitalisierung des Lernens und den damit verbundenen Veränderungen der Bedingungen pädagogischer Kommunikation weiterhin vollzogen werden kann. Theorien, in deren Grundlegung Ungewissheit eine zentrale Rolle spielt, erweisen sich demnach als erkenntnisreiche Anknüpfungspunkte, die es empirisch weiter auszuloten gilt.

Funding Open Access funding enabled and organized by Projekt DEAL.

Open Access Dieser Artikel wird unter der Creative Commons Namensnennung 4.0 International Lizenz veröffentlicht, welche die Nutzung, Vervielfältigung, Bearbeitung, Verbreitung und Wiedergabe in jeglichem Medium und Format erlaubt, sofern Sie den/die ursprünglichen Autor(en) und die Quelle ordnungsgemäß nennen, einen Link zur Creative Commons Lizenz beifügen und angeben, ob Änderungen vorgenommen wurden.

Die in diesem Artikel enthaltenen Bilder und sonstiges Drittmaterial unterliegen ebenfalls der genannten Creative Commons Lizenz, sofern sich aus der Abbildungslegende nichts anderes ergibt. Sofern das betreffende Material nicht unter der genannten Creative Commons Lizenz steht und die betreffende Handlung nicht nach gesetzlichen Vorschriften erlaubt ist, ist für die oben aufgeführten Weiterverwendungen des Materials die Einwilligung des jeweiligen Rechteinhabers einzuholen.

Weitere Details zur Lizenz entnehmen Sie bitte der Lizenzinformation auf http://creativecommons.org/licenses/by/4.0/deed.de.

\section{Literatur}

Amann, K., \& Hirschauer, S. (1997). Die Befremdung der eigenen Kultur. Zur ethnographischen Herausforderung soziologischer Empirie. Frankfurt am Main: Suhrkamp.

Breidenstein, G. (2017). Interdisziplinäre Tradition und disziplinäre Konvention in der erziehungswissenschaftlichen Ethnographie. Zeitschrift für Qualitative Forschung, 18(1), 9-20.

Breidenstein, G., \& Kelle, H. (1998). Geschlechteralltag in der Schulklasse. Ethnographische Studien zur Gleichaltrigenkultur. Weinheim, München: Juventa.

Breidenstein, G., \& Kelle, H. (2002). Die Schulklasse als Publikum. Zum Verhältnis von peer culture und Unterricht. Die Deutsche Schule, 94(3), 318-329.

Breidenstein, G., Hirschauer, S., Kalthoff, H., \& Nieswand, B. (2013). Ethnografie. Die Praxis der Feldforschung. Stuttgart: UTB.

Cafantaris, K. (2020). Umgang mit Wissen in der Kulturarbeit für Erwachsene. Pädagogische Kommunikation in hybriden Settings der Erwachsenenbildung. Wiesbaden: Springer VS.

Cafantaris, K. (2021). Ethnographie als Interaktionsprozess - Zur ethnographischen Untersuchung hybrider Lernorte. ZfW, 44, 5. https://doi.org/10.1007/s40955-021-00178-3.

Cloos, P. (2010). Narrative Beobachtungsprotokolle. In F. Heinzel, W. Thole, P. Cloos \& S. Köngeter (Hrsg.), „Auf unsicherem Terrain “. Ethnographische Forschung im Kontext des Bildungs- und Sozialwesens (S. 181-191). Wiesbaden: VS.

Dinkelaker, J. (2008). Kommunikation von (Nicht-)Wissen. Eine Fallstudie zum Lernen Erwachsener in hybriden Settings. Wiesbaden: VS.

Dinkelaker, J. (2018). Lernen Erwachsener. Stuttgart: Kohlhammer.

Geertz, C. (1987). Dichte Beschreibung. Beiträge zum Verstehen kultureller Systeme. Frankfurt am Main: Suhrkamp.

Goffman, E. (1959). Wir alle spielen Theater. München: Piper.

Helsper, W. (2008). Ungewissheit und pädagogische Professionalität. In Bielefelder Arbeitsgruppe 8 (Hrsg.), Soziale Arbeit in Gesellschaft (Bd. 8, S. 155-161). Wiesbaden: VS. 
Hünersdorf, B. (2009). Der klinische Blick in der sozialen Arbeit. Systemtheoretische Annäherungen an eine Reflexionstheorie des Hilfesystems. Wiesbaden: VS.

Kade, J. (2004). Erziehung als pädagogische Kommunikation. In D. Lenzen (Hrsg.), Irritationen des Erziehungssystems. Pädagogische Resonanzen auf die Systemtheorie (S. 199-232). Frankfurt am Main: Suhrkamp.

Kade, J. (2017). Subjektivierungsformen und Subjektivierungspraktiken. Zur Ordnung des Pädagogischen diesseits und jenseits der Schule. In EEO - Enzyklopädie Erziehungswissenschaft Online. Weinheim: Beltz Juventa.

Kade, J., \& Seitter, W. (2007). Pädagogische Kommunikation. Bd. 1. Opladen, Farmington Hills: Barbara Budrich.

Kieserling, A. (1999). Kommunikation unter Anwesenden. Frankfurt am Main: Suhrkamp.

Lindner, R. (1981). Die Angst des Forschers vor dem Feld. Überlegungen zur teilnehmenden Beobachtung als Interaktionsprozeß. Zeitschrift für Volkskunde, 77, 51-66.

Lüders, C., Kade, J., \& Hornstein, W. (2004). Entgrenzung des Pädagogischen. In W. Hornstein (Hrsg.), Einführung in Grundbegriffe und Grundfragen der Erziehungswissenschaft (S. 223-232). Wiesbaden: Springer.

Luhmann, N. (2000). Organisation und Entscheidung. Opladen, Wiesbaden: Westdeutscher Verlag.

Luhmann, N. (2005). Soziologische Aufklärung 6. Die Soziologie und der Mensch (2. Aufl.). Wiesbaden: VS.

Meseth, W., Proske, M., \& Radtke, F.-O. (2011). Was leistet eine kommunikationstheoretische Modellierung des Gegenstandes „Unterricht“. In W. Meseth, M. Proske \& F.-O. Radtke (Hrsg.), Unterrichtstheorien in Forschung und Lehre (S. 223-240). Bad Heilbrunn: Klinkhardt.

Parsons, T., \& Shils, E. (1951). Towards a general theory of action. Cambridge: American Sociological Association.

Paseka, A., Keller-Schneider, M., \& Combe, A. (Hrsg.). (2018). Ungewissheit als Herausforderung für pädagogisches Handeln. Wiesbaden: Springer VS.

Schulz, M. (2014). Ethnografische Beobachtung. In A. Tillmann, S. Fleischer \& K. U. Hugger (Hrsg.), Handbuch Kinder und Medien (S. 225-235). Wiesbaden: Springer VS. https://doi.org/10. 1007/978-3-531-18997-0_17.
Seitter, W. (1999). Riskante Übergänge in der Moderne. Vereinskulturen Bildungsbiographien, Migranten. Wiesbaden: VS.

Seitter, W. (2000). Lesen, Vereinsmeiern, Reisen. (Vergessene) Elemente einer Theorie lebenslangen Lernens. Zeitschrift für Pädagogik, 46(1), 81-96.

Seitter, W. (2011). Pädagogische Felder. In J. Kade, W. Helsper, C. Lüders, B. Egloff, F.-O. Radtke \& W. Thole (Hrsg.), Pädagogisches Wissen. Erziehungswissenschaft in Grundbegriffen (S. 8-92). Stuttgart: Kohlhammer.

Stürmer, S., \& Siem, B. (2020). Sozialpsychologie der Gruppe. München: Ernst Reinhard Verlag.

Vonneilich, N. (2020). Soziale Beziehungen, soziales Kapital und soziale Netzwerke - eine begriffliche Einordnung. In A. Klärner, M. Gamper, S. Keim - Klärner, I. Moor, H. von der Lippe \& N. Lippe Vonneilich (Hrsg.), Soziale Netzwerke und gesundheitliche Ungleichheiten. Wiesbaden: Springer VS. https://doi.org/10. 1007/978-3-658-21659-7_2.

Wilde, D. (2015). Dinge sammeln. Annäherung an eine Kulturtechnik. Bielefeld: transcript.

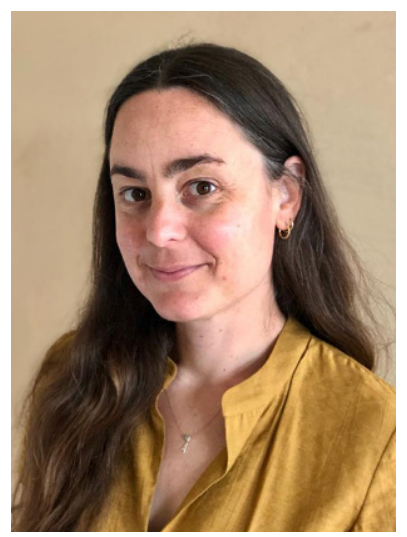

Dr. Karola Cafantaris ist wissenschaftliche Mitarbeiterin (PostDoc) an der Helmut-Schmidt Universität-Hamburg. Ihre Arbeitsschwerpunkte sind Erziehungswissenschaftliche Ethnographie, Unterrichtsforschung, Heterogenität sowie Digitälität in Schule und Unterricht. 